\title{
Role and Regulation of Poly-3-Hydroxybutyrate in Nitrogen Fixation in Azorhizobium caulinodans
}

\author{
Nick Crang, ${ }^{1}$ Khushboo Borah, ${ }^{1}$ Euan K. James, ${ }^{2}$ Beatriz Jorrín, ${ }^{1}$ Patrick Green, ${ }^{1}$ Andrzej Tkacz, ${ }^{1}$ \\ Alison K. East, ${ }^{1}$ and Philip S. Poole ${ }^{1+}$ \\ ${ }^{1}$ Department of Plant Sciences, University of Oxford, South Parks Road, Oxford, OX1 3RB, U.K. \\ ${ }^{2}$ The James Hutton Institute, Invergowrie, Dundee DD2 5DA, U.K.
}

Accepted 10 August 2021.

\begin{abstract}
An Azorhizobium caulinodans phaC mutant (OPS0865) unable to make poly-3-hydroxybutyrate (PHB), grows poorly on many carbon sources and cannot fix nitrogen in laboratory culture. However, when inoculated onto its host plant, Sesbania rostrata, the phaC mutant consistently fixed nitrogen. Upon reisolation from $S$. rostrata root nodules, a suppressor strain (OPS0921) was isolated that has significantly improved growth on a variety of carbon sources and also fixes nitrogen in laboratory culture. The suppressor retains the original mutation and is unable to synthesize PHB. Genome sequencing revealed a suppressor transition mutation, $\mathrm{G}$ to $\mathrm{A}$ (position 357,354), 13 bases upstream of the ATG start codon of phaR in its putative ribosome binding site (RBS). PhaR is the global regulator of PHB synthesis but also has other roles in regulation within the cell. In comparison with the wild type, translation from the phaR native RBS is increased approximately sixfold in the phaC mutant background, suggesting that the level of PhaR is controlled by PHB. Translation from the phaR mutated RBS (RBS*) of the suppressor mutant strain (OPS0921) is locked at a low basal rate and unaffected by the phaC mutation, suggesting that RBS* $^{*}$ renders the level of PhaR insensitive to regulation by PHB. In the original phaC mutant (OPS0865), the lack of nitrogen fixation and poor growth on many carbon sources is likely to be due to increased levels of PhaR causing dysregulation of its complex regulon, because PHB formation, per se, is not required for effective nitrogen fixation in $\boldsymbol{A}$. caulinodans.
\end{abstract}

Keywords: Azorhizobium caulinodans, bacteria-plant symbiosis, carbon sink, PhaR, PHB, poly-3-hydroxybutyrate, reductant storage, Rhizobium-legume symbiosis, Sesbania rostrata

Biological nitrogen fixation by diazotrophic bacteria enables them to convert atmospheric dinitrogen into ammonia using the enzyme nitrogenase (Oldroyd et al. 2011). Azorhizobium caulinodans, a species of the order Rhizobiales, is able to fix nitrogen under free-living conditions, as well as during symbiosis with its host plant, Sesbania rostrata (Dreyfus et al. 1983, 1988;

${ }^{\dagger}$ Corresponding author: P. S. Poole; philip.poole@plants.ox.ac.uk

Funding: This work was supported by the Biotechnology and Biological Sciences Research Council grant numbers BB/M011224/1 and BB/ N013387/1.

The author(s) declare no conflict of interest. distributed under the CC BY 4.0 International license.
Elmerich et al. 1982). This is unusual for nitrogen-fixing rhizobia, because most can only fix nitrogen when in symbiosis with their host plant (Terpolilli et al. 2016).

Given that the nitrogenase enzyme is permanently inactivated by oxygen, a low-oxygen environment is essential for successful nitrogen fixation to occur (Rutten and Poole 2019). This presents a problem, because rhizobia, including $A$. caulinodans, are typically obligate aerobic organisms using the tricarboxylic acid (TCA) cycle as their primary means of energy generation. Under low-oxygen conditions, there may be insufficient oxygen to act as a terminal acceptor for electron transport to balance reductant formation. One of the main ways to remove an excess of reductant is channeling reductant into the generation of carbon storage molecules such as lipids or poly-3-hydroxybutyrate (PHB) (Terpolilli et al. 2016).

PHB is a carbon storage molecule synthesized by most rhizobia (Trainer and Charles 2006) using molecules of acetyl CoA in a multistage reaction, the polymerization step of which is catalyzed by PHB synthase (PhaC) (Schubert et al. 1988; Sharma and Dhingra 2016) (Fig. 1). In addition to its role as a reductant sink, the carbon storage provided by PHB has been shown to support nitrogen fixation during periods of carbon starvation and it has been suggested that is has a role in initial nodule formation (Gerson et al. 1978; Wang et al. 2007). Many species of rhizobia, including A. caulinodans, Bradyrhizobium japonicum, Rhizobium etli, and $R$. tropici, accumulate PHB both under freeliving conditions (Stam et al. 1986; Tombolini and Nuti 1989) and within the nitrogen-fixing nodule form of rhizobia known as bacteroids (Karr et al. 1984; Ndoye et al. 1994).

Mutagenesis studies performed in a number of species to determine the importance of PHB in nitrogen fixation have produced a mixture of results. In $R$. etli, phaC mutants fix nitrogen at higher levels than the wild type (Cevallos et al. 1996), whereas reduced symbiotic efficiency was seen in Sinorhizobium meliloti in symbiosis with Medicago truncatula but not with M. sativa (Wang et al. 2007). No change was seen in the fixation rates of phaC mutants of $R$. leguminosarum in nodules on Pisum sativum or Phaseolus vulgaris (Lodwig et al. 2005).

Formation of PHB is regulated by the interaction of PhaR, an autoregulating negative regulator (Maehara et al. 2002), and phasin proteins (PhaP). Phasins are present in both prokaryotes and archaea (Cai et al. 2015; Jang et al. 2017; Kim et al. 2017), where they are sequestered to the surface of polyhydroxyalkanoate granules such as $\mathrm{PHB}$, reducing the apparent concentration within the cytoplasm despite continuous synthesis. When the granules reach a critical size, PhaP displaces PhaR from the surface due to the greater binding affinity to PHB of PhaP relative to PhaR. This results in a buildup of cytoplasmic PhaR, which then binds to the promoters of phaR, 
phaP, and phaC, preventing their transcription and halting production of further PHB (Kuchta et al. 2007; Pötter et al. 2002).

Previous knockout mutations of phaC showed it to be essential for nitrogen fixation and effective growth in A. caulinodans, suggesting that PHB synthesis is required for fixation in this species (Mandon et al. 1998). During symbiosis, this mutant strain was found to primarily produce small, nonfixing nodules containing no bacteria; however, it was noted at the time that some plants also contained "a few large nitrogen-fixing nodules", which were theorized to be revertants (Mandon et al. 1998). In this work, we confirm that an A. caulinodans phaC mutant cannot fix nitrogen in the laboratory but consistently forms suppressor strains in planta that recover $\mathrm{N}_{2}$ fixation. $\mathrm{PHB}$ synthesis, per se, is unnecessary for effective fixation by A. caulinodans in either free-living or symbiotic conditions. Instead, phaR dysregulation prevents $\mathrm{N}_{2}$ fixation and reduces growth of a phaC mutant.

\section{RESULTS}

Phenotypic characterization of phaC mutants.

An A. caulinodans phaC mutant strain (OPS0865) was isolated via single integration using the plasmid pK19mob. Under free-living conditions, OPS0865 did not fix nitrogen (Fig. 2A). Additionally, at $21 \%$ oxygen, the phaC mutant had reduced growth on acetate, arabinose, and pyruvate, though growth on succinate was unaffected (Fig. 3A). This growth defect was even more severe at $1 \%$ oxygen (Fig. 3B). Growth on succinate had the shortest generation time and was equivalent in the mutant and wild type at $21 \%$ oxygen (approximately $3.2 \pm 0.1$ h). However, when grown on succinate at $1 \%$ oxygen, the phaC mutant had a generation time of $19.3 \pm 1.5 \mathrm{~h}$, compared with $7.5 \pm 1.2 \mathrm{~h}$ for wild-type ORS571. Thus, mutation of phaC disrupts growth of $A$. caulinodans and this disruption is exacerbated by oxygen limitation. Several attempts to complement the mutation with a plasmid-borne copy of phaC failed. Therefore, to determine whether the mutation in phaC causes the observed phenotype, we isolated 12 revertants, where the integrated pK19mob in phaC was deleted, by repeated growth on media lacking antibiotics. Revertants were screened for restored sensitivity to neomycin and the presence of a full-length phaC gene using primers oxp3394 and oxp3395. All 12 revertants were restored to wild-type growth on tryptone-yeast (TY) media, and 1 such revertant strain (OPS2077) was retained for further analysis. Wild-type levels of growth on all carbon sources were

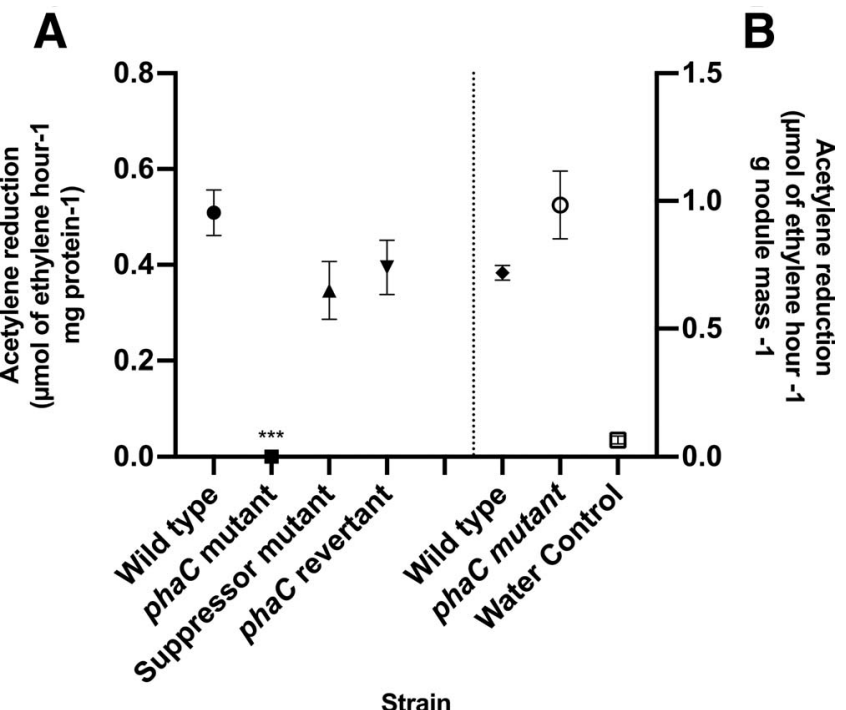

Fig. 2. Acetylene reduction activity (ARA) under free-living and symbiotic nitrogen-fixing conditions for wild-type ORS571, the phaC mutant (OPS0865), suppressor mutant (OPS0921), and phaC revertant (OPS2077) strains of Azorhizobium caulinodans. A, Mean ARA of free-living bacteria in micromoles of ethylene per hour per milligram of protein. The three strains ORS571, OPS0921, and OPS2077 are significantly different from strain OPS0865 $(P<0.005)$, Mann-Whitney test; $n=6$. B, Mean ARA during symbiosis at 28 days postinoculation of Sesbania rostrata in micromoles of ethylene per hour per gram of nodule. Water control $=$ plants inoculated with water alone. No significant difference was observed between the wild type and the phaC mutant $(P=0.4)$, two-tailed Mann-Whitney test. Error bars show standard error; $n=3$.

A

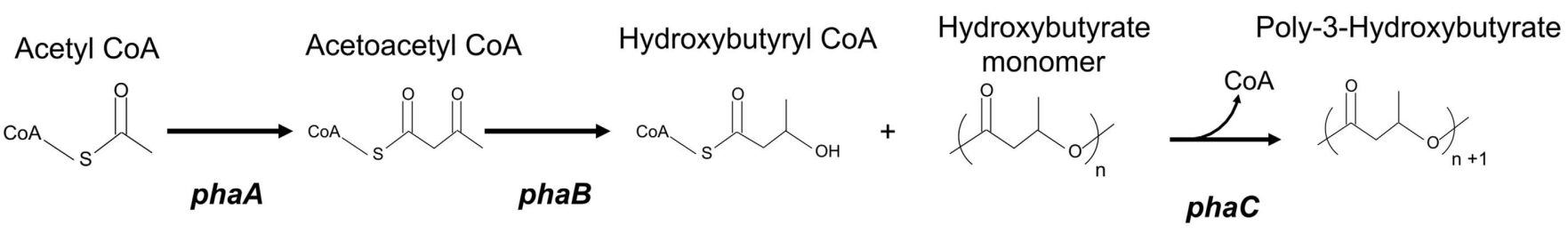

B

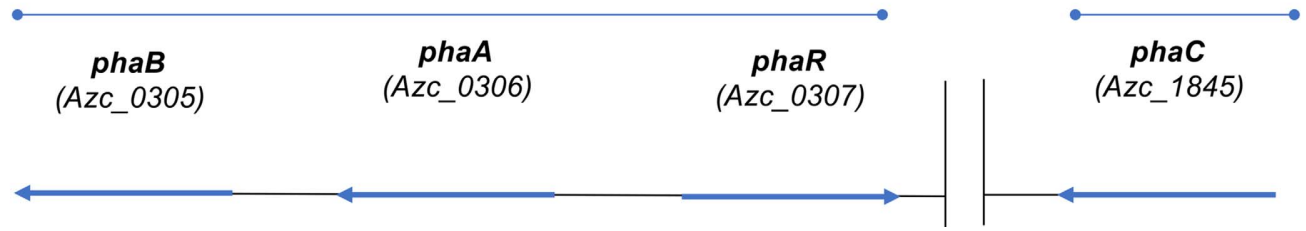

Fig. 1. Poly-3-hydroxybutyrate (PHB) synthesis in Azorhizobium caulinodans and the genes encoding the proteins involved. A, Enzymic reactions in PHB synthesis. Condensation of two acetyl CoA molecules by PhaA to acetoacetyl CoA, followed by reduction to hydroxybutyryl CoA by PhaB. Monomeric hydroxybutyrate is linked to hydroxybutyryl $\mathrm{CoA}$ by $\mathrm{PhaC}$ to form the polymer, releasing CoA. This polymer is extended by PhaC-catalyzed reactions with additional hydroxybutyryl CoA. B, Genome architecture of genes encoding the proteins involved in PHB synthesis. Gene numbers and absolute genome positions for the two regions are indicated. PhaA is an acetyl CoA acetyl-transferase, PhaB is an acetoacetyl CoA reductase, PhaC is a PHB synthase, and PhaR is a regulator. 
restored in the revertant strain (Fig. 3) along with wild-type rates of free-living nitrogen fixation (Fig. 2A). These observations are in line with previous reports (Mandon et al. 1998) that PHB is required for effective growth on a variety of carbon sources and alters $\mathrm{N}_{2}$ fixation in A. caulinodans.

To our surprise, when the phaC mutant was inoculated onto Sesbania rostrata plants, we observed wild-type symbiotic nitrogen fixation rates on all plants measured; furthermore, all nodules on the plants were large and had a pink, wild-type appearance (Fig. 2B). Our initial suspicion was that these fixation rates were due to a spontaneous reversion mutant being favored by the plant, resulting in the loss of the phaC mutation and causing the wild-type appearance of the nodules. To test whether a such a mutation had occurred, bacteria were isolated from six separate nodules from plants inoculated with the phaC mutant. These colonies were initially isolated on TY without added antibiotics and individual purified colonies were then screened for neomycin resistance and the presence of the pK19mob plasmid, using PCR detection with a chromosomal primer (oxp1043) and a pK19mob-specific primer (pK19B), amplifying outwards from the plasmid backbone. All purified colonies retained neomycin resistance, while PCR amplification gave a $1.5-\mathrm{kb}$ fragment in both the original phaC mutant strain (OPS0865) and in the nodule-derived isolates but gave no band in the wild type. Glycerol stocks were made of three PCRmapped nodule isolates (OPS0921, OPS0922, and OPS0923). Wild-type levels of free-living nitrogen fixation were observed in free-living cultures of the nodule-isolated strain OPS0921, whereas the original phaC mutant retained a $\mathrm{Fix}^{-}$phenotype (Fig. 2A). Thus, passage through the plant restored nitrogen fixation to the phaC mutant strain. Because these nodule isolated strains (OPS0921, OPS0922, and OPS0923) retained the original phaC mutation, we subsequently refer to them as suppressor strains.

Quantification of PHB and growth of phaC mutants.

To check for the presence of $\mathrm{PHB}$, we made transmission electron micrographs of $S$. rostrata nodules. White, electron-
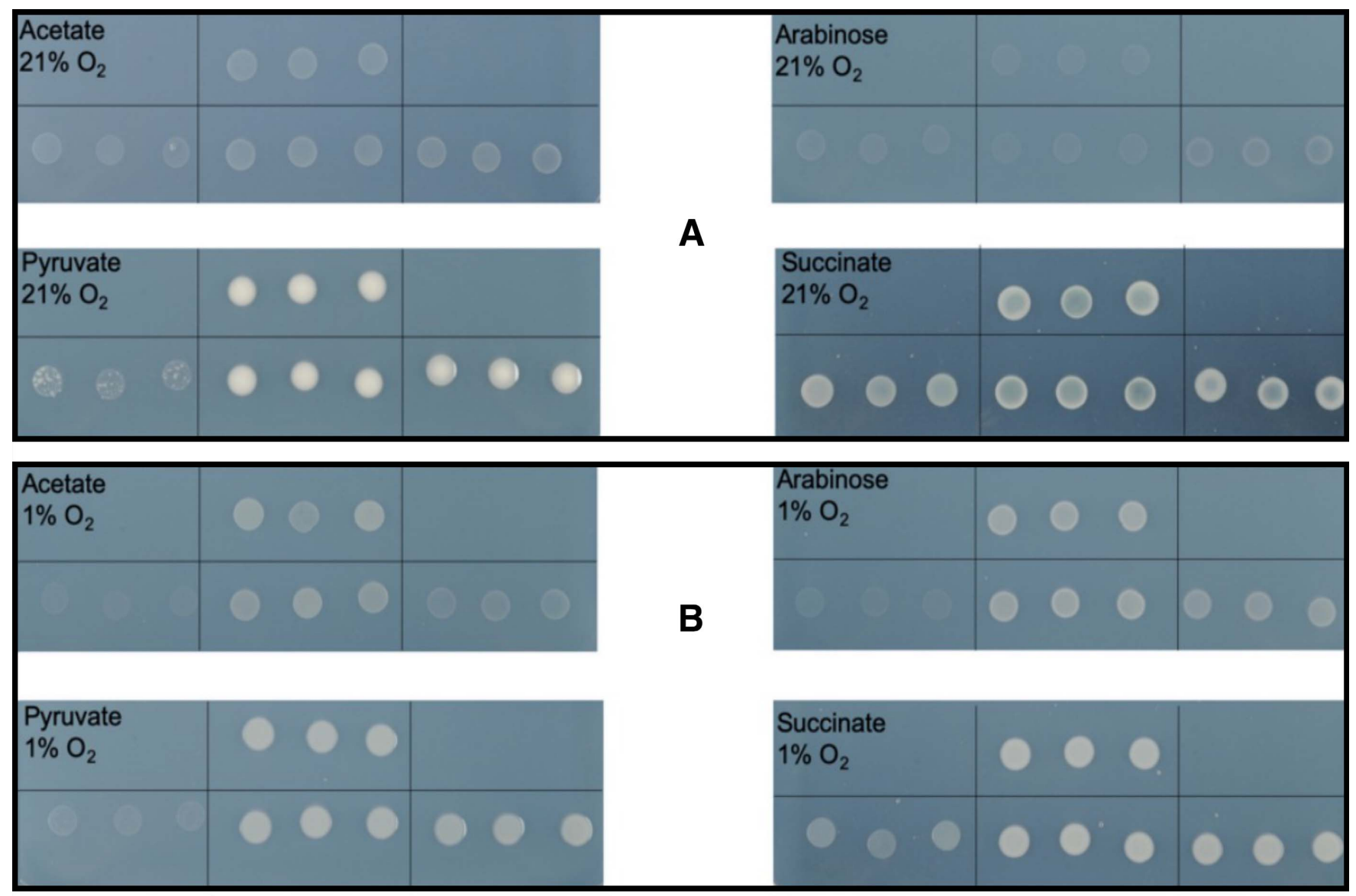

C

\begin{tabular}{|c|c|c|}
\hline $\begin{array}{c}\text { Sole carbon source } \\
\mathrm{O}_{2} \text { concentration }\end{array}$ & Wild type (ORS571) & \\
\hline phaC mutant (OPS0865) & $\begin{array}{c}\text { phaC reversion mutant } \\
\text { (OPS2077) }\end{array}$ & $\begin{array}{c}\text { phaC suppressor mutant } \\
\text { (OPS0921) }\end{array}$ \\
\hline
\end{tabular}

Fig. 3. Growth of the wild type (ORS571), phaC mutant (OPS0865), phaC revertant (OPS2077), and suppressor mutant (OPS0921) strains of Azorhizobium caulinodans at $28^{\circ} \mathrm{C}$ on acetate, arabinose, pyruvate, or succinate as a sole carbon source. A, At $21 \%$ oxygen; $\mathbf{B}$, at $1 \%$ oxygen; and $\mathbf{C}$, relative position of bacterial strains. Bacteria $(5 \mu \mathrm{l}$ at an optical density at $600 \mathrm{~nm}$ of approximately 0.1$)$ were drop plated onto solid media with sole carbon source shown; $n=3$. 
transparent PHB granules were present in the wild type (Fig. 4A) but neither in the phaC mutant (OPS0865) nor the putative phaC suppressor mutant (OPS0921) (Fig. 4).

Because the phaC mutant and the putative suppressor mutant OPS0921 had such a clear difference in their ability to fix nitrogen, their growth was assessed at both 21 and $1 \%$ oxygen to identify any differences (Fig. 3). The phaC mutant showed the biggest reduction in growth relative to the wild type, with the putative suppressor (OPS0921) producing an intermediate growth phenotype. Of particular note was the poor growth of the phaC mutant on acetate and pyruvate as sole carbon sources at both oxygen tensions.

Given the altered growth of the putative suppressor strain (OPS0921), the retention of neomycin resistance and pK19mob insertion, together with the lack of PHB synthesis, it was apparent that, in addition to the original phaC mutation, there must be a suppressor mutation present in this strain. To identify the mutation, whole-genome sequencing was undertaken utilizing the sequencing services of MicrobesNG. The three isolated putative suppressor strains (OPS0921, OPS0922, and OPS0923) were sequenced and compared with both the phaC mutant and the wild type, with the assumption that any mutation shared by the putative suppressor strains (OPS0921, 0922 and 0923) but absent from the original phaC mutant and ORS571 may cause the suppression. As expected, the draft genomes of all putative suppressors (OPS0921, 0922, and 0923) retained the pK19mob plasmid backbone. However, there is a singlenucleotide polymorphism (SNP) present in all three putative suppressor mutants (OPS0921, OPS0922, and OPS0923) and absent from both the wild type and the original phaC mutant. This is a G-to-A transition (position 357,354), 13 bases upstream of the phaR ATG start codon. The location of the SNP is likely to be within the ribosome binding site (RBS) of the phaR gene and, thus, may affect translation of phaR. Because the three putative suppressor strains were otherwise genetically identical, further investigations were carried out on the strain OPS0921 alone, hereafter referred to as the suppressor mutant. Hereafter, this SNP will be referred to as 0921 SNP and this mutated RBS as RBS*.

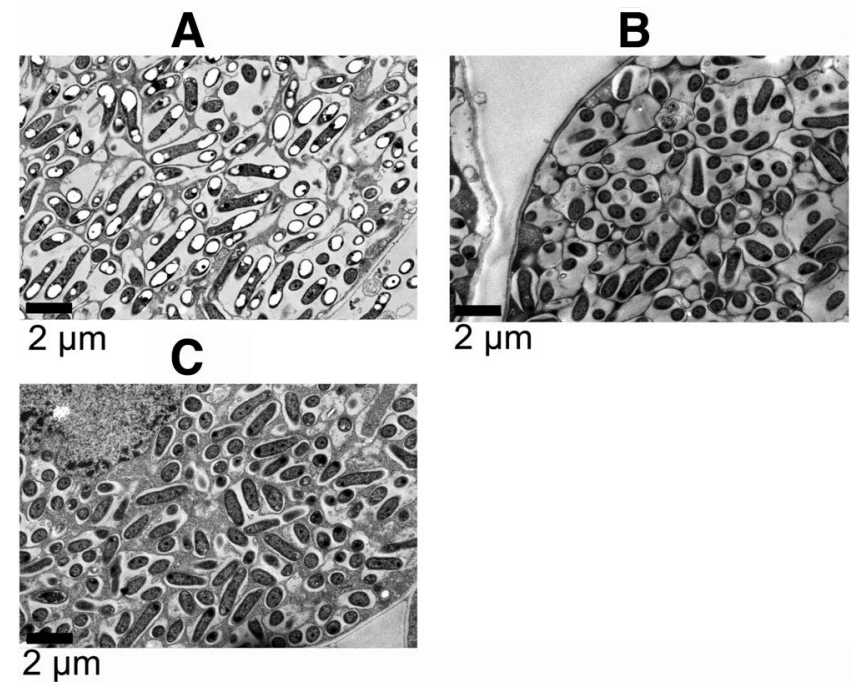

Fig. 4. Transmission electron micrographs showing bacteroids within sections from nodules of Sesbania rostrata at 28 days postinoculation. Poly-3-hydroxybutyrate (PHB) granules are white inclusions within bacteroids A, Wild type (ORS571) in which PHB granules are clearly visible; B, phaC mutant (OPS0865), no PHB visible; and C, phaC suppressor mutant (OPS0921), no PHB visible. Scale bars are shown.
Translation and transcription of phaR

in different strain backgrounds.

Translation of phaR was measured using translational fusions to green fluorescent protein (GFP). The native phaR RBS fusion produced sixfold more GFP in the phaC mutant than in that of the wild type (Fig. 5A). This increase in GFP translation (and by extension, PhaR protein) was likely to be a response to the lack of PHB in the phaC mutant strain. Thus, translation of phaR is reduced by the presence of PHB and, consequently, in the absence of PHB, the level of PhaR increases. In contrast, the phaR RBS* translational fusion gave a consistently low GFP signal in both the phaC and wild-type ORS571 backgrounds (Fig. 5A), suggesting that RBS* is insensitive to the presence of PHB in the bacterial cell. Thus, the RBS* 0921 SNP mutation has locked translation of phaR at a low level independent of PHB.

Transcription of phaR and phaA was measured in the wild type and the phaC mutant by quantitative reverse-transcription PCR (qRT-PCR) (using gyrA as a control housekeeping gene). There was no significant difference between the two strains in the transcription of either phaR or phaA (Fig. 5B), consistent with the effect of the suppressor mutations being at the translational level.

\section{DISCUSSION}

The phaC mutant has a reduced growth rate on acetate, arabinose, and pyruvate at atmospheric oxygen levels but not on succinate (Fig. 3A). This was not unexpected, given the high levels of acetyl-CoA generated by growth on these three carbon sources, and is in line with previous PHB mutants in $R$. etli and R. leguminosarum bv. viciae 3841 (Cevallos et al. 1996; Lodwig et al. 2005). The observed growth defect was far greater at low oxygen (1\%), with reduced growth even on succinate. This limited growth is likely to result from flux imbalance, with acetate and pyruvate generating acetyl-CoA directly, which feeds into the TCA cycle. This generates $4 \mathrm{~mol}$ of reductant for every 1 mol of pyruvate utilized. Succinate, by contrast, can generate small amounts of reductant via malic enzyme, or malate dehydrogenase activity, and can be converted to sugar via gluconeogenesis. This sugar can be converted to storage molecules or catabolized and fed into the TCA cycle at a more controlled rate. As a result, the phaC mutants grown on succinate displayed the smallest growth defect. The wild-type strain and the revertant strain (OPS2077) do not display any growth impairment on acetate or pyruvate because they can use PHB as an acetyl-CoA and reductant sink. Thus, under a low oxygen concentration, synthesis of PHB helps to maintain redox balance in A. caulinodans. Although growth of $A$. caulinodans strains on arabinose also showed the importance of $\mathrm{PHB}$ at $1 \%$ oxygen, growth was unexpectedly but consistently better at 1 than $21 \%$.

Although the phaC mutant did not fix nitrogen in free-living conditions (Fig. 2A), phaC suppressor mutants (e.g., OPS0921), capable of nitrogen fixation, were consistently isolated from root nodules of $S$. rostrata (Fig. 2B). Transmission electron microscopy (TEM) micrographs (Fig. 4) confirmed that the suppressor strains lacked PHB; thus, its absence does not prevent nitrogen fixation in A. caulinodans. Whole-genome sequencing showed that the suppressor strains have a mutation in the putative ribosome binding site of phaR. GFP translational fusions to phaR demonstrated that the wild-type RBS had a sixfold increase in translation in the phaC mutant compared with the wild type, while the mutated RBS from the suppressor strains (RBS*) had a uniformly low translation rate in both wild-type and phaC backgrounds (Fig. 5). PhaR levels are usually regulated by PHB levels within the cell (Maehara et al. 2002). The location of the SNP within the RBS of the major regulatory gene phaR in the 
suppressor strain suggests lack of PHB results in the dysregulation of PhaR. In B. japonicum, such a mutation would presumably result in the downregulation of more than 200 genes, including the key regulator fix $K_{2}$ (Quelas et al. 2016). This downregulation, combined with the lack of the major reductant sink PHB, would explain the extremely poor growth rate of the phaC mutant strain (OPS0865). The partial rescue of growth in the suppressor strain (OPS0921) appears to have been achieved by reducing translation of phaR to basal levels (Figs. 3 and 5A). This appears to prevent some of the effects of PhaR dysregulation; however, growth rates were not restored to wild-type levels, probably due to the suppressor stain lacking PHB as a reductant sink. The dysregulation of PhaR levels may also explain why attempts to rescue the initial phaC mutant strain with plasmid-borne copies of the phaC gene were unsuccessful, because multiple copies of phaC may also result in PhaR dysregulation as a result of excess $\mathrm{PHB}$ production, though this remains speculation.

It was shown by qRT-PCR that there was no significant difference in transcription of either phaR or phaA in either the wild type or the phaC mutant (Fig. 5B). The lack of a significant change in phaA transcription in the phaC mutant strain is surprising given the sixfold increase in translation in this strain of the repressor PhaR. We would have expected to observe a much greater reduction in phaA transcription, and this suggests that any reduction in expression caused directly by $\mathrm{PhaR}$ may be modest. The numerous genes encoding regulatory proteins whose expression is controlled by $\mathrm{PhaR}$ (e.g., fixk2) could serve to amplify the effect of this modest reduction (Quelas et al. 2016), contributing to the reduced growth seen in the phaC mutant strain compared with the suppressor strain (Fig. 3).

Because a reduced level of the global $\mathrm{PhaR}$ regulator restored wild-type nitrogen fixation in suppressor strains of phaC (Fig. 2A), this suggests that the loss of fixation was due to an excess of PhaR rather than the lack of a PHB as a reductant sink. This brings A. caulinodans in line with most other rhizobial species, which are able to fix nitrogen without PHB synthesis (Cevallos et al. 1996; Lodwig et al. 2005; Quelas et al. 2013) by making use of other reductant sinks such as lipid or, potentially, glycogen (Lodwig et al. 2005; Wang et al. 2007).

Overall, it is clear that, while PHB is not strictly necessary for nitrogen fixation in A. caulinodans, it has an important role both as a redox sink and for maintaining the level of PhaR in the cell. Disruption of either of these roles can result in a severe growth defect, particularly when grown on carbon sources that result in high levels of acetyl-CoA.

\section{MATERIALS AND METHODS}

Bacterial strains, media, and bacterial growth conditions.

All bacterial strains, plasmids, and primers used in this study are listed in Table 1. A. caulinodans strains were routinely grown on solid media at $37^{\circ} \mathrm{C}$ on TY (Beringer 1974) or universal minimal salts (UMS) with $1 \times$ stock vitamins (thiamine hydrochloride at $1 \mathrm{~g} \mathrm{liter}^{-1}$, D-pantothenic acid calcium salt at $2 \mathrm{~g} \mathrm{liter}^{-1}$, and Biotin at $100 \mathrm{mg} \mathrm{liter}^{-1}$ ); $300 \mu \mathrm{M}$ nicotinic acid; either 10 $\mathrm{mM}$ glutamate or $10 \mathrm{mM}$ ammonium chloride (as a nitrogen source); and $20 \mathrm{mM}$ succinate, $6 \mathrm{mM}$ acetate, $20 \mathrm{mM}$ arabinose, or $30 \mathrm{mM}$ pyruvate (as a carbon source). UMS is a modified version of ammonium mineral salt medium (Poole et al. 1994), with alterations previously published (Pini et al. 2017). Experiments using $A$. caulinodans liquid cultures were grown at $28^{\circ} \mathrm{C}$ (this was the maximum temperature that could be maintained by the oxygen cabinet which was used for acetylene reduction activity [ARA] experiments with free-living bacteria). Antibiotics in A. caulinodans were used at the following concentrations, unless stated otherwise; ampicillin $(100 \mu \mathrm{g} / \mathrm{ml})$, gentamycin $(25 \mu \mathrm{g} / \mathrm{ml})$ neomycin $(80 \mu \mathrm{g} / \mathrm{ml})$, and nitrofurantoin $(5 \mu \mathrm{g} / \mathrm{ml})$. Escherichia coli strains were grown at $37^{\circ} \mathrm{C}$ in Luria-Bertani medium using gentamycin $(10 \mu \mathrm{g} / \mathrm{ml})$ and kanamycin $(20 \mu \mathrm{g} / \mathrm{ml})$.

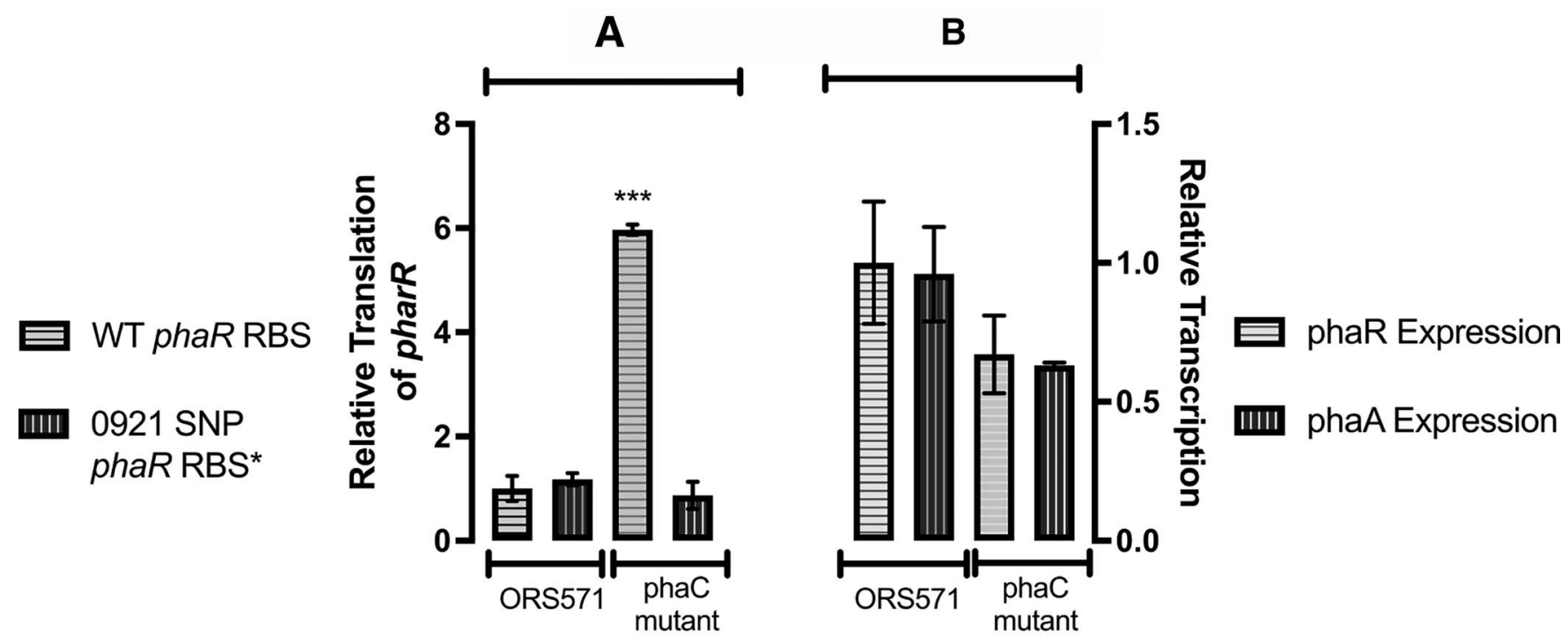

\section{Strain}

Fig. 5. Translation and transcription of phaR and transcription of phaA in the wild type (WT) (ORS571) and phaC mutant (OPS0865) backgrounds of free-living Azorhizobium caulinodans. A, Relative translation from phaR ribosome binding site (RBS) (WT) and phaR RBS* (SNP 0921) measured by fluorescence of a green fluorescent protein (GFP) translational reporter. Data are relative to GFP measured from phaR RBS (WT) in WT (ORS571) background. Error bars are standard error. Tukey's multiple comparison test indicated a significant difference between RBS and RBS* in phaC mutant (OPS0865) backgrounds; asterisks $(* * *)$ indicate $P<0.0001$, degrees of freedom $=89$. No significant difference is seen between any combination of the other three samples; $n=8$. B, Relative transcription measured by quantitative reverse-transcription PCR of phaA and phaR in WT (ORS571) and phaC mutant (OPS0865) strains, compared with housekeeping gene gyrA. Error bars are standard error. No significant difference was seen between expression of these genes in either strain; $n=3$. 
Table 1. Strains, plasmids, and primer sequences used in this study

\begin{tabular}{|c|c|c|}
\hline Strains, plasmids, and primers & Relevant characteristics and primer sequence $^{a}$ & Source \\
\hline \multicolumn{3}{|l|}{ Strain } \\
\hline Azorhizobium caulinodans ORS571 & Wild type, ampicillin resistant & Dreyfus et al. 1983 \\
\hline OPS0865 & $\begin{array}{l}\text { phaC mutant generated by integration of pOPS } 0391 \text { into } \\
\text { ORS571, ampicillin resistant, neomycin resistant }\end{array}$ & This study \\
\hline OPS0921 & $\begin{array}{l}\text { Suppressor mutant isolated from nitrogen-fixing root nodules } \\
\text { of Sesbania rostrata inoculated with OPS0865, ampicillin } \\
\text { resistant, neomycin resistant }\end{array}$ & This study \\
\hline OPS0922 & $\begin{array}{l}\text { Suppressor mutant isolated from nitrogen-fixing root nodules } \\
\text { of } S \text {. rostrata inoculated with OPS0865, ampicillin } \\
\text { resistant, neomycin resistant }\end{array}$ & This study \\
\hline OPS0923 & $\begin{array}{l}\text { Suppressor mutant isolated from nitrogen-fixing root nodules } \\
\text { of } S \text {. rostrata inoculated with OPS0865, ampicillin } \\
\text { resistant, neomycin resistant }\end{array}$ & This study \\
\hline OPS1426 & $\begin{array}{l}\text { pOPS } 1029 \text { conjugated into ORS571, ampicillin resistant, } \\
\text { gentamycin resistant }\end{array}$ & This study \\
\hline OPS1427 & $\begin{array}{l}\text { pOPS } 1030 \text { conjugated into ORS571, ampicillin resistant, } \\
\text { gentamycin resistant }\end{array}$ & This study \\
\hline OPS1432 & $\begin{array}{l}\text { pOPS } 1029 \text { conjugated into OPS0865, ampicillin resistant, } \\
\text { gentamycin resistant }\end{array}$ & This study \\
\hline OPS1433 & $\begin{array}{l}\text { pOPS } 1030 \text { conjugated into OPS } 0865 \text {, ampicillin resistant, } \\
\text { gentamycin resistant }\end{array}$ & This study \\
\hline OPS1438 & $\begin{array}{l}\text { pOPS } 1029 \text { conjugated into OPS0921, ampicillin resistant, } \\
\text { gentamycin resistant }\end{array}$ & This study \\
\hline OPS1439 & $\begin{array}{l}\text { pOPS } 1030 \text { conjugated into OPS0921, ampicillin resistant, } \\
\text { gentamycin resistant }\end{array}$ & This study \\
\hline OPS2077 & $\begin{array}{l}\text { OPS0865 reversion mutant which has lost the integrated } \\
\text { pk19mob via multiple sub culturing on TY, confirmed via } \\
\text { PCR across the phaC (PHB synthase) gene with } \\
\text { oxp3394 }+3395(1,900 \text { bp in wild type) }\end{array}$ & This study \\
\hline Escherichia coli DH5 $\alpha$ & 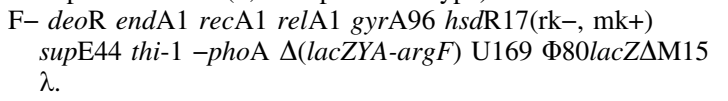 & Bioline \\
\hline E. coli 803 & $\begin{array}{l}\text { hsdS (rk-, mk-, gal-, met-) in this work contained pRK2013 } \\
\text { helper plasmid }\end{array}$ & Wood 1996 \\
\hline \multicolumn{3}{|c|}{${ }^{1}$} \\
\hline pK19mob & $\begin{array}{l}\text { Mobilizable } E \text {. coli cloning vector, kanamycin and neomycin } \\
\text { resistant }\end{array}$ & Schäfer et al. 1994 \\
\hline pOPS0391 & $\begin{array}{l}1.1 \mathrm{~kb} \text { of ORS571 phaC generated by PCR with oxp1218/ } \\
1219 \text { InFusion cloned into HindIII-linearized pK19mob, } \\
\text { kanamycin and neomycin resistant }\end{array}$ & This study \\
\hline pOPS1029 & $\begin{array}{l}\text { phaC-phaR intergenic region }+90 \text { bp of phaR (generated by } \\
\text { PCR with oxp } 2419 / 2420 \text { from wild type ORS571 genomic } \\
\text { DNA (RBS) InFusion cloned into pRU1097 linearized by } \\
\text { PCR with oxp } 2421 / \text { oxp } 2422 \text {, gentamycin resistant }\end{array}$ & This study \\
\hline pOPS1030 & $\begin{array}{l}\text { phaC-phaR intergenic region }+90 \text { bp of phaR (generated by } \\
\text { PCR with oxp } 2419 / 2420 \text { from suppressor mutant OPS } 0921 \\
\text { genomic DNA (RBS*) InFusion cloned into pRU1097 } \\
\text { linearized by PCR with oxp2421/oxp2422, gentamycin } \\
\text { resistant }\end{array}$ & This study \\
\hline pRK2013 & $\begin{array}{l}\text { ColEI replicon with RK2 tra genes; helper plasmid used for } \\
\text { mobilizing plasmids, kanamycin resistant }\end{array}$ & Karunakaran et al. 2005 \\
\hline pRU1097 & $\begin{array}{l}\text { p318/p319 PCR product (gfpmut3.1) from pBJA27 cloned in } \\
\text { pOT2 as SpeI-SacI promoter-less GFP module, gentamycin } \\
\text { resistant }\end{array}$ & Karunakaran et al. 2005 \\
\hline \multicolumn{3}{|c|}{ 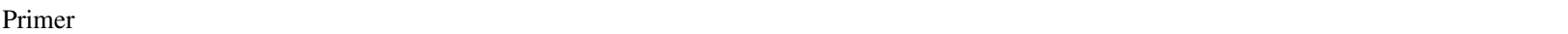 } \\
\hline p318 & $\begin{array}{l}\text { GAGAGAGAACTAGTGGAGGAAGAAAAAATGCG- } \\
\text { TAAAGGAGAAGAACTTTTCA }\end{array}$ & Karunakaran et al. 2005 \\
\hline P319 & CTCTCGAGCTCATTTGTATAGTTCATCCATGC & Karunakaran et al. 2005 \\
\hline M13 forward & $\begin{array}{l}\text { CACGACGTTGTAAAACGA; sequencing primer for insert } \\
\text { cloned in pK19mob }\end{array}$ & Bahar et al. 1998 \\
\hline M13 reverse & $\begin{array}{l}\text { GGATAACAATTTCACACAGG; sequencing primer for } \\
\text { insert cloned in pK19mob }\end{array}$ & Bahar et al. 1998 \\
\hline oxp1043 & $\begin{array}{l}\text { TTTTTGGTACCATGGAGGCGTTCGCCCAGAA; forward } \\
\text { mapping primer for phaC gene }\end{array}$ & This study \\
\hline oxp1218 & $\begin{array}{l}\text { TGATTACGCCAAGCTATAAGCGGAGTTGGGCGG; } \\
\text { forward PCR primer to amplify internal phaC fragment }\end{array}$ & This study \\
\hline oxp1219 & $\begin{array}{l}\text { GCAGGCATGCAAGCTGTTCTTCGACGCCCTGAAGC; } \\
\text { reverse PCR primer to amplify internal phaC fragment }\end{array}$ & This study \\
\hline oxp2419 & $\begin{array}{l}\text { GTGGAGGAAGAAAAATGGGCTTCTCTCCTGGGG; } \\
\text { forward PCR primer to amplify phaC-phaR intergenic } \\
\text { region }+90 \text {-bp phaR }\end{array}$ & This study \\
\hline
\end{tabular}

(Continued on next page)

${ }^{a}$ Primer sequences are presented $5^{\prime}$ to $3^{\prime}$. PHB $=$ poly-3-hydroxybutyrate, RBS = ribosome binding site, GFP = green fluorescent protein, and qRT-PCR = quantitative reverse-transcription PCR. 
Table 1. (Continued from previous page)

\begin{tabular}{|c|c|c|}
\hline Strains, plasmids, and primers & Relevant characteristics and primer sequence ${ }^{a}$ & Source \\
\hline oxp2420 & $\begin{array}{l}\text { TTCTTCTCCTTTACGTTCGAGGGTCACATAGGTACTG; } \\
\text { reverse PCR primer to amplify phaC-phaR intergenic } \\
\text { region + 90-bp phaR }\end{array}$ & This study \\
\hline oxp2421 & $\begin{array}{l}\text { CGTAAAGGAGAAGAACTTTTCACTGG; forward inverse } \\
\text { PCR primer to linearize pRU1097 }\end{array}$ & This study \\
\hline oxp2422 & $\begin{array}{l}\text { TTTTTCTTCCTCCACTAGTCTCTCT; reverse inverse PCR } \\
\text { primer to linearize pRU1097 }\end{array}$ & This study \\
\hline oxp2475 & $\begin{array}{l}\text { GAACGGCTACGAGTGGAAC; forward qRT-PCR primer } \\
\text { for gyrA }\end{array}$ & This study \\
\hline oxp2476 & $\begin{array}{l}\text { GGAGAAGGTCTGCACCATG; reverse qRT-PCR primer for } \\
\text { gyrA }\end{array}$ & This study \\
\hline oxp2971 & $\begin{array}{l}\text { CCATGTTGCGGCGAACC; forward qRT-PCR primer for } \\
\text { phaR }\end{array}$ & This study \\
\hline oxp2972 & $\begin{array}{l}\text { CGCTTCCTCGAGATGTCCAT; reverse qRT-PCR primer } \\
\text { for phaR }\end{array}$ & This study \\
\hline oxp2976 & $\begin{array}{l}\text { CCATGTGGTAGCCGTTGAAG; reverse qRT-PCR primer } \\
\text { for phaA }\end{array}$ & This study \\
\hline oxp3105 & $\begin{array}{l}\text { ATGAGCCAGTCCACCCATG; forward qRT-PCR primer for } \\
\text { phaA }\end{array}$ & This study \\
\hline oxp3394 & $\begin{array}{l}\text { TGCCGAATTCGGATCCGGAGAAGCTGACGGCGC- } \\
\text { GAAA; forward PCR mapping primer phaC }\end{array}$ & This study \\
\hline oxp3395 & $\begin{array}{l}\text { TCAACAGGAGTCCAAGAGCGCGATTAAGGCAGGTGA- } \\
\text { CAGC; reverse PCR mapping primer phaC }\end{array}$ & This study \\
\hline pk19A & $\begin{array}{l}\text { ATCAGATCTTGATCCCCTGC; PCR mapping primer for } \\
\text { pK19mob }\end{array}$ & Karunakaran et al. 2010 \\
\hline
\end{tabular}

Testing bacterial growth on different carbon sources.

Single colonies of A. caulinodans strains were grown overnight in $\mathrm{TY}$ at $37^{\circ} \mathrm{C}$, then diluted to an optical density at 600 $\mathrm{nm}\left(\mathrm{OD}_{600}\right)$ of approximately 0.1 . Three biological replicates of each strain were pipetted as $5 \mu$ l-droplets onto UMS agar plates supplemented with vitamins, nicotinic acid, ammonium chloride, and either acetate, arabinose, pyruvate, or succinate as the sole carbon source. Plates were incubated at 21 or $1 \%$ oxygen at $28^{\circ} \mathrm{C}$ for $60 \mathrm{~h}$ before being photographed.

\section{Growth and nodulation of $S$. rostrata.}

Seed were sterilized for $10 \mathrm{~min}$ in concentrated sulfuric acid to break seed dormancy, then washed in $400 \mathrm{ml}$ of sterile MilliQ water. Seed were germinated for 3 days on $2 \%$ water agar containing a double layer of autoclaved filter paper soaked in MilliQ water. Germinated seed were planted individually in 1-liter pots containing autoclaved vermiculite and $300 \mathrm{ml}$ of nitrogen-free rooting solution $\left(4 \mathrm{mM} \mathrm{Na} \mathrm{HPO}_{4}, 3.7 \mathrm{mM} \mathrm{K} \mathrm{PO}_{4}, 1 \mathrm{mM}\right.$ $\mathrm{CaCl}_{2}, 800 \mu \mathrm{M} \mathrm{MgSO}_{4}, 100 \mu \mathrm{M} \mathrm{KCl}, 35 \mu \mathrm{M} \mathrm{H}_{3} \mathrm{BO}_{3}, 10 \mu \mathrm{M} \mathrm{Fe}$ EDTA, $9 \mu \mathrm{M} \mathrm{MnCl}_{2}, 0.8 \mu \mathrm{M} \mathrm{ZnCl} 2,0.5 \mu \mathrm{M} \mathrm{Na} \mathrm{MoO}_{4}$, and $0.3 \mu \mathrm{M} \mathrm{CuSO}_{4}$ ). Inoculum was prepared from a TY slope (containing ampicillin or neomycin), grown for 3 days at $37^{\circ} \mathrm{C}$ by resuspending the bacteria in $3 \mathrm{ml}$ of TY. Inoculum $(1 \mathrm{ml}$, diluted 1/100) was applied to each germinated seed. Four germinating seeds were inoculated with each bacterial strain. Plants were watered with sterile water every 2 days and grown at $22^{\circ} \mathrm{C}$ for 28 days in a controlled-environment room with a photoperiod of $16 \mathrm{~h}$ of light and $8 \mathrm{~h}$ of darkness.

\section{Generation of a phaC mutant.}

A. caulinodans mutants were generated using integration through homologous recombination (single cross-over) into the genome of plasmid DNA cloned in the mobilizable vector pK19mob (Schäfer et al. 1994). Plasmid pOPS0391 was constructed by InFusion (Takara Bio) cloning $1.1 \mathrm{~kb}$ of ORS571 phaC gene amplified with primers oxp1218 and oxp1219 into the HindIII site of pK19mob. Construction of pOPS0391 was confirmed by sequencing with primers M13 forward and M13 reverse (Eurofins Genomics). Conjugation of pOPS0391 into ORS571 was performed using a donor strain of $E$. coli
(pOPS0391) and E. coli strain 803 (pRK2013). The conjugation mix was allowed to grow overnight on $\mathrm{TY}$ agar at $37^{\circ} \mathrm{C}$, then counter-selected using neomycin $(240 \mu \mathrm{g} / \mathrm{ml})$ and nitrofurantoin. The mutation was confirmed by PCR mapping with primers oxp1043 and pK19A and sequencing the amplified fragment (Eurofins Genomics).

\section{Reversion of phaC mutation.}

A single colony of the phaC mutant was grown for $16 \mathrm{~h}$ in 5 $\mathrm{ml}$ of TY liquid culture with no antibiotics, before being subcultured into fresh TY and grown for a further $16 \mathrm{~h}$. On the following day, $20 \mu \mathrm{l}$ of culture was plated onto TY ampicillin and incubated for 2 days at $37^{\circ} \mathrm{C}$. Colonies were patched onto TY neomycin and TY ampicillin agar plates. Neomycin-sensitive cultures were screened for the presence of phaC by PCR using oxp3394 and oxp3395. An isolate which has lost the pK19mob insertion in the chromosome and has a full-length phaC gene was retained as the reversion strain (OPS2077).

\section{Assessing translation with GFP reporters.}

GFP translational fusions to phaR gene of the wild type (RBS) and the suppressor mutant (RBS*) strains were made by PCR amplification with oxp2419 and oxp2420 (amplifying the phaA:phaR intergenic region from ORS571 and OPS0921, together with $90 \mathrm{bp}$ of phaR gene). The products were cloned into pRU1097 (linearized by inverse PCR with oxp2421 and oxp2422) by InFusion HD cloning (Takara Bio). These constructs were conjugated into the A. caulinodans wild type (ORS571), phaC mutant (OPS0865), and suppressor mutant (OPS0921) backgrounds. Cultures were grown overnight in $10 \mathrm{ml}$ of UMS containing $10 \mathrm{mM}$ glutamate as the added nitrogen source. Cultures were grown to an $\mathrm{OD}_{600}$ of approximately 0.4 before $200-\mu \mathrm{l}$ aliquots were measured in triplicate for GFP fluoresce (excitation at $485 \mathrm{~nm}$, absorbance at $520 \mathrm{~nm}$ ) and $\mathrm{OD}_{600}$ in a 96-well plate using a FLUOstar Omega spectrophotometric plate reader.

\section{Assessing transcription by qRT-PCR.}

RNA was extracted from $10-\mathrm{ml} A$. caulinodans TY cultures using the Direct-zol miniprep kit (Zymo). RNA was DNase treated using a Turbo DNA-free kit (Invitrogen). RNA 
concentration was assessed via a Qubit RNA HS Assay Kit (Thermo Fisher Scientific) and converted to cDNA using Superscript II (Invitrogen). Housekeeping gene gyrA was used as a reference. qRT-PCR was performed using a SensiMix SYBR No-ROX Kit (Bioline) according to the manufacturer's instructions on a StepOnePlus qRT-PCR machine (Applied Biosystems).

\section{Bioinformatic analysis.}

Genomes of the phaC mutant and the three putative suppressor strains (OPS0921, OPS0922, and OPS0923) were sequenced by MicrobesNG (Birmingham, U.K.) service using Illumina Miseq 300PE technology. Reads from the phaC mutant and putative suppressor mutants (OPS0921, OPS0922, and OPS0923) were aligned against the chromosome of $A$. caulinodans ORS571 (NCBI: txid438753) using Bowtie2 v2.1.0 in "very-sensitive" parameters in end-to-end mode (Langmead and Salzberg 2012). The output of the alignment was transformed using SAMtools v1.4 (Li et al. 2009) and SNP detected with VarScan v2.4.3 (Koboldt et al. 2013).

\section{Free-living A. caulinodans ARA.}

Cultures were prepared by streaking single colonies of bacteria on $10-\mathrm{ml}$ UMS slopes ( $0.6 \%$ agarose) in $28-\mathrm{ml}$ universal tubes, supplemented with succinate, vitamins, nicotinic acid, and $10 \mathrm{mM} \mathrm{NH} \mathrm{NH}_{4}$. After 2 days of incubation at $37^{\circ} \mathrm{C}$, cultures were washed from the slopes three times with phosphatebuffered saline to remove residual nitrogen and resuspended in $2 \mathrm{ml}$ of nitrogen-free UMS media at $\mathrm{OD}_{600 \mathrm{~nm}}=0.4$ in universals. Cultures were placed in a controlled-atmosphere cabinet adjusted to $3 \% \mathrm{O}_{2}$ by flushing with $\mathrm{N}_{2}$ gas, left for $1 \mathrm{~h}$ with shaking at $180 \mathrm{rpm}$, and sealed with a rubber top. To initiate fixation, $10 \%$ of the air $(3.35 \mathrm{ml})$ from the headspaces of universals was removed with a syringe and replaced with $\mathrm{C}_{2} \mathrm{H}_{2}(2.8 \mathrm{ml})$. Cultures were then returned to incubation at $37^{\circ} \mathrm{C}$ and $180 \mathrm{rpm}$ for $4 \mathrm{~h}$. The production of $\mathrm{C}_{2} \mathrm{H}_{4}$ from $\mathrm{C}_{2} \mathrm{H}_{2}$ was measured at $4 \mathrm{~h}$ and again at $6 \mathrm{~h}$ postinitiation using gas chromatography mass spectrometry (Clarus 480 gas chromatograph; Perkin Elmer) and the rates of nitrogenase activity were subsequently calculated between these two time points. The protein concentrations of the cell lysates were quantified using a bicinchoninic acid protein assay (Sigma-Aldrich). Six biological replicates were included for each strain measured. Fixation rates are given in micromoles of ethylene per hour per milligram of protein.

\section{In planta A. caulinodans ARA.}

ARA was measured according to (Trinick et al. 1976), except $2 \%$ acetylene was used and incubation was for $2 \mathrm{~h}$ at room temperature before measurement on a gas chromatogram (Perkin Elmer). Fixation rates are given in micromoles of ethylene per hour per gram of nodule.

\section{TEM of sectioned nodules.}

The procedure used was modified from that of Beck et al. (2008) in the following manner: a JEOL JEM1400 transmission electron microscope was used, samples were not hexane treated, and pyroxylin-coated grids were used to collect sections cut on a Leica UCT ultramicrotome.

\section{Data and materials availability.}

All data and materials generated are available from P. S. Poole upon request.

\section{LITERATURE CITED}

Bahar, M., de Majnik, J., Wexler, M., Fry, J., Poole, P. S., and Murphy, P. J. 1998. A model for the catabolism of rhizopine in Rhizobium leguminosarum involves a ferredoxin oxygenase complex and the inositol degradative pathway. Mol. Plant-Microbe Interact. 11: 1057-1068.

Beck, S., Marlow, V. L., Woodall, K., Doerrler, W. T., James, E. K., and Ferguson, G. P. 2008. The Sinorhizobium meliloti MsbA2 protein is essential for the legume symbiosis. Microbiol. Read. 154:1258-1270.

Beringer, J. E. 1974. R factor transfer in Rhizobium leguminosarum. J. Gen. Microbiol. 84:188-198.

Cai, S., Cai, L., Zhao, D., Liu, G., Han, J., Zhou, J., and Xiang, H. 2015. A novel DNA-binding protein, PhaR, plays a central role in the regulation of polyhydroxyalkanoate accumulation and granule formation in the haloarchaeon Haloferax mediterranei. Appl. Environ. Microbiol. 81:373-385.

Cevallos, M. A., Encarnación, S., Leija, A., Mora, Y., and Mora, J. 1996. Genetic and physiological characterization of a Rhizobium etli mutant strain unable to synthesize poly-beta-hydroxybutyrate. J. Bacteriol 178:1646-1654.

Dreyfus, B., Garcia, J. L., and Gillis, M. 1988. Characterization of Azorhizobium caulinodans gen. nov., sp. nov., a stem-nodulating nitrogenfixing bacterium isolated from Sesbania rostrata. Int. J. Syst. Bacteriol. 38:89-98.

Dreyfus, B. L., Elmerich, C., and Dommergues, Y. R. 1983. Free-living Rhizobium strain able to grow on $\mathrm{n}(2)$ as the sole nitrogen source Appl. Environ. Microbiol. 45:711-713.

Elmerich, C., Dreyfus, B. L., Reysset, G., and Aubert, J. P. 1982. Genetic analysis of nitrogen fixation in a tropical fast-growing Rhizobium. EMBO J. 1:499-503.

Gerson, T., Patel, J. J., and Wong, M. N. 1978. The effects of age, darkness and nitrate on poly- $\beta$-hydroxybutyrate levels and nitrogen-fixing ability of Rhizobium in Lupinus angustifolius. Physiol. Plant. 42:420-424.

Jang, S. H., Jang, H. A., Lee, J., Kim, J. U., Lee, S. A., Park, K. E., Kim, B. H., Jo, Y. H., and Lee, B. L. 2017. PhaR, a negative regulator of PhaP, modulates the colonization of a Burkholderia gut symbiont in the midgut of the host insect, Riptortus pedestris. Appl. Environ. Microbiol. 83:e00459-17.

Karr, D. B., Waters, J. K., Suzuki, F., and Emerich, D. W. 1984. Enzymes of the poly- $\beta$-hydroxybutyrate and citric acid cycles of $R h i$ zobium japonicum bacteroids. Plant Physiol. 75:1158-1162.

Karunakaran, R., Haag, A. F., East, A. K., Ramachandran, V. K., Prell, J., James, E. K., Scocchi, M., Ferguson, G. P., and Poole, P. S. 2010 BacA is essential for bacteroid development in nodules of galegoid, but not phaseoloid, legumes. J. Bacteriol. 192:2920-2928.

Karunakaran, R., Mauchline, T. H., Hosie, A. H. F., and Poole, P. S. 2005. A family of promoter probe vectors incorporating autofluorescent and chromogenic reporter proteins for studying gene expression in Gram-negative bacteria. Microbiol. Read. 151:3249-3256.

Kim, Y., Lee, K., Kim, H., Cho, S., and Lee, J. 2017. Inhibition of poly 3-hydroxybutyrate (PHB) synthesis by phaR deletion in Methylobacterium extorquens AM1. Korean Chem. Eng. Res. 55:363-368.

Koboldt, D. C., Larson, D. E., and Wilson, R. K. 2013. Using VarScan 2 for germline variant calling and somatic mutation detection. Curr. Protoc. Bioinf. 44:15.4.1-15.4.17.

Kuchta, K., Chi, L., Fuchs, H., Pötter, M., and Steinbüchel, A. 2007. Studies on the influence of phasins on accumulation and degradation of PHB and nanostructure of PHB granules in Ralstonia eutropha H16. Biomacromolecules 8:657-662.

Langmead, B., and Salzberg, S. L. 2012. Fast gapped-read alignment with Bowtie 2. Nat. Methods 9:357-359.

Li, H., Handsaker, B., Wysoker, A., Fennell, T., Ruan, J., Homer, N., Marth, G., Abecasis, G., Durbin, R., and 1000 Genome Project Data Processing Subgroup. 2009. The Sequence Alignment/Map format and SAMtools. Bioinformatics 25:2078-2079.

Lodwig, E. M., Leonard, M., Marroqui, S., Wheeler, T. R., Findlay, K., Downie, J. A., and Poole, P. S. 2005. Role of polyhydroxybutyrate and glycogen as carbon storage compounds in pea and bean bacteroids. Mol. Plant-Microbe Interact. 18:67-74.

Maehara, A., Taguchi, S., Nishiyama, T., Yamane, T., and Doi, Y. 2002. A repressor protein, PhaR, regulates polyhydroxyalkanoate (PHA) synthesis via its direct interaction with PHA. J. Bacteriol. 184:3992-4002.

Mandon, K., Michel-Reydellet, N., Encarnación, S., Kaminski, P. A., Leija, A., Cevallos, M. A., Elmerich, C., and Mora, J. 1998. Poly-betahydroxybutyrate turnover in Azorhizobium caulinodans is required for growth and affects nifA expression. J. Bacteriol. 180:5070-5076.

Ndoye, I., de Billy, F., Vasse, J., Dreyfus, B., and Truchet, G. 1994. Root nodulation of Sesbania rostrata. J. Bacteriol. 176:1060-1068.

Oldroyd, G. E. D., Murray, J. D., Poole, P. S., and Downie, J. A. 2011. The rules of engagement in the legume-rhizobial symbiosis. Annu. Rev. Genet. 45:119-144. 
Pini, F., East, A. K., Appia-Ayme, C., Tomek, J., Karunakaran, R., Mendoza-Suárez, M., Edwards, A., Terpolilli, J. J., Roworth, J., Downie, J. A., and Poole, P. S. 2017. Bacterial biosensors for in vivo spatiotemporal mapping of root secretion. Plant Physiol. 174:1289-1306.

Poole, P. S., Schofield, N. A., Reid, C. J., Drew, E. M., and Walshaw, D. L. 1994. Identification of chromosomal genes located downstream of $\operatorname{dct} D$ that affect the requirement for calcium and the lipopolysaccharide layer of Rhizobium leguminosarum. Microbiol. Read. 140:2797-2809.

Pötter, M., Madkour, M. H., Mayer, F., and Steinbüchel, A. 2002. Regulation of phasin expression and polyhydroxyalkanoate (PHA) granule formation in Ralstonia eutropha H16. Microbiol. Read. 148:2413-2426.

Quelas, J. I., Mesa, S., Mongiardini, E. J., Jendrossek, D., and Lodeiro, A. R. 2016. Regulation of polyhydroxybutyrate synthesis in the soil bacterium Bradyrhizobium diazoefficiens. Appl. Environ. Microbiol. 82:4299-4308.

Quelas, J. I., Mongiardini, E. J., Pérez-Giménez, J., Parisi, G., and Lodeiro, A. R. 2013. Analysis of two polyhydroxyalkanoate synthases in Bradyrhizobium japonicum USDA 110. J. Bacteriol. 195:3145-3155.

Rutten, P. J., and Poole, P. S. 2019. Oxygen regulatory mechanisms of nitrogen fixation in rhizobia. Adv. Microb. Physiol. 75:325-389.

Schäfer, A., Tauch, A., Jäger, W., Kalinowski, J., Thierbach, G., and Pühler, A. 1994. Small mobilizable multi-purpose cloning vectors derived from the Escherichia coli plasmids pK18 and pK19: Selection of defined deletions in the chromosome of Corynebacterium glutamicum. Gene 145:69-73.

Schubert, P., Steinbüchel, A., and Schlegel, H. G. 1988. Cloning of the Alcaligenes eutrophus genes for synthesis of poly-beta-hydroxybutyric acid (PHB) and synthesis of PHB in Escherichia coli. J. Bacteriol. 170:5837-5847.
Sharma, M., and Dhingra, H. 2016. Poly- $\beta$-hydroxybutyrate: A biodegradable polyester, biosynthesis and biodegradation. Br. Microbiol. Res. J. 14:1-11.

Stam, H., van Verseveld, H. W., de Vries, W., and Stouthamer, A. H. 1986. Utilization of poly- $\beta$-hydroxybutyrate in free-living cultures of Rhizobium ORS571. FEMS Microbiol. Lett. 35:215-220.

Terpolilli, J. J., Masakapalli, S. K., Karunakaran, R., Webb, I. U., Green, R., Watmough, N. J., Kruger, N. J., Ratcliffe, R. G., and Poole, P. S 2016. Lipogenesis and redox balance in nitrogen-fixing pea bacteroids. J. Bacteriol. 198:2864-2875.

Tombolini, R., and Nuti, M. P. 1989. Poly( $\beta$-hydroxyalkanoate) biosynthesis and accumulation by different Rhizobium species. FEMS Microbiol. Lett. 60:299-304.

Trainer, M. A., and Charles, T. C. 2006. The role of PHB metabolism in the symbiosis of rhizobia with legumes. Appl. Microbiol. Biotechnol. 71:377-386.

Trinick, M. J., Dilworth, M. J., and Grounds, M. 1976. Factors affecting the reduction of acetylene by root nodules of Lupinus species. New Phytol. 77:359-370.

Wang, C., Saldanha, M., Sheng, X., Shelswell, K. J., Walsh, K. T., Sobral, B. W. S., and Charles, T. C. 2007. Roles of poly-3-hydroxybutyrate (PHB) and glycogen in symbiosis of Sinorhizobium meliloti with Medicago sp. Microbiol. Read. 153:388-398.

Wood, W. B. 1996. Host specificity of DNA produced by Escherichia coli: Bacterial mutations affecting the restriction and modification of DNA J. Mol. Biol. 16:118-133. 